\title{
Symmetric strong vector quasiequilibrium problems in Hausdorff locally convex spaces
}

\author{
Bin Chen ${ }^{1}$, Nan-jing Huang ${ }^{1}$ and Yeol Je Cho ${ }^{2^{*}}$
}

\author{
* Correspondence: yjcho@gnu.ac.kr \\ ${ }^{2}$ Department of Mathematics \\ Education and the RINS, \\ Gyeongsang National University, \\ Chinju 660-701, Korea \\ Full list of author information is \\ available at the end of the article
}

\begin{abstract}
In this article, a new symmetric strong vector quasiequilibrium problem in real locally convex Hausdorff topological vector spaces is introduced and studied. An existence theorem of solutions for the symmetric strong vector quasiequilibrium problem by using Kakutani-Fan-Glicksberg fixed point theorem is obtained. Moreover, the closedness of the solution set for this problem is derived. The results presented in this article improve and extend some known results according to Long et al. [Math. Comput. Model. 47, 445-451 (2008)], Somyot and Kanokwan [Fixed Point Theory Appl. doi:10.1155/2011/475121], and Wang et al. [Bull. Malays. Math. Sci. Soc. http:// www.emis.de/journals/BMMSS/pdf/acceptedpapers/2009-11-022_R1.pdf].

2000 MSC: 49J40; 90C29.
\end{abstract}

Keywords: symmetric strong vector quasiequilibrium problem, Kakutani-Fan-Glicksberg fixed point theorem, closedness

\section{Introduction}

Equilibrium problem was introduced by Blum and Oettli [1] (see, also Noor and Oettli [2]). It provides a unified model of several classes of problems, for example, optimization problems, problems of Nash equilibrium, fixed point problems, variational inequalities, and complementarity problems. In recent years, there has been an increasing interest in the study of vector equilibrium problems. A lot of results for existence of solutions for vector equilibrium problems and vector variational inequalities have been established by many authors in different ways. For details, we refer the reader to various studies (see, e.g., [3-15] and the references therein).

Let $X$ and $Z$ be real locally convex Hausdorff spaces, $K \subset X$ be a nonempty subset, and $C \subset Z$ be a closed convex pointed cone. Let $F: K \times K \rightarrow 2^{Z}$ be a given set-valued mapping. Ansari et al. [16] introduced two types of set-valued vector equilibrium problems as follows. The first type is the weak vector equilibrium problem: finding $x \in K$ such that

$$
F(x, y) \not \subset-\text { int } C, \quad \forall y \in K \text {. }
$$

The second type is the strong vector equilibrium problem (SVEP): finding $x \in K$ such that

$$
F(x, y) \subset C, \quad \forall y \in K
$$

\section{Springer}

(c) 2011 Chen et al; licensee Springer. This is an Open Access article distributed under the terms of the Creative Commons Attribution License (http://creativecommons.org/licenses/by/2.0), which permits unrestricted use, distribution, and reproduction in any medium, provided the original work is properly cited. 
If int $C \neq \varnothing$ and $x$ satisfies (1.1), then we call $x$ a weak efficient solution for the vector equilibrium problem. If $x$ satisfies (1.2), then we call $x$ a strong efficient solution for the vector equilibrium problem.

It is worth mentioning that many existing results of the vector equilibrium problem are obtained under the assumption that the dual $C^{*}$ of the ordering cone $C$ has a $w_{e a k^{*}}$ compact base. As we know, for a normed space, the dual cone $C^{*}$ has a weak* compact base if and only if int $C \neq \varnothing$ (see [17]). However, in many cases, the ordering cone has an empty interior. For example, in the classical Banach spaces $l^{p}$ and $L^{p}(\Omega)$, where $1<p<\infty$, the standard ordering cone has an empty interior. Thus, it is interesting for the study of the existence of solutions and the properties of the solution sets for this case.

On the other hand, it is well known that a strong efficient solution of vector equilibrium problem is an ideal solution. It is better than other solutions, such as efficient solution, weak efficient solution, proper efficient solution, and supper efficient solution (see [18]). Hence, it is important to study the existence of strong efficient solution and the properties of the strong efficient solution set. Very recently, Long et al. [19] gave an existence theorem for generalized strong vector quasiequilibrium problem (GSVQEP) and discussed the stability of strong efficient solutions. Somyot and Kanokwan [20] derived an existence theorem for system of generalized strong vector quasiequilibrium problem (SGSVQEP) which extends the main results of Long et al. [19].

Motivated and inspired by the research studies mentioned above, in this article, we consider a type of symmetric strong vector quasiequilibrium problem (SSVQEP) without assuming that the dual of the ordering cone has a weak* compact base. Let $X, Y$, and $Z$ be real locally convex Hausdorff topological vector spaces, $K \subset X$ and $D \subset Y$ be nonempty subsets, and $C \subset Z$ be a nonempty closed convex cone. Suppose that $S_{1}, S_{2}$ : $K \times K \rightarrow 2^{K}, T_{1}, T_{2}: K \times K \rightarrow 2^{D}$ and $F_{1}, F_{2}: K \times D \times K \rightarrow 2^{Z}$ are set-valued mappings. We consider the following SSVQEP: finding $(\bar{x}, \bar{u}) \in K \times K$ and $\bar{y} \in T_{1}(\bar{x}, \bar{u})$, $\bar{v} \in T_{2}(\bar{x}, \bar{u})$ such that $\bar{x} \in S_{1}(\bar{x}, \bar{u}), \bar{u} \in S_{2}(\bar{x}, \bar{u})$,

$$
F_{1}(\bar{x}, \bar{y}, z) \subset C, \quad \forall z \in S_{1}(\bar{x}, \bar{u})
$$

and

$$
F_{2}(\bar{u}, \bar{v}, z) \subset C, \quad \forall z \in S_{2}(\bar{x}, \bar{u}) .
$$

We call this $(\bar{x}, \bar{u})$ a strong efficient solution for the SSVQEP.

In this article, we establish an existence theorem of the solutions for the SSVQEP by using Kakutani-Fan-Glicksberg fixed point theorem. We also discuss the closedness of the solution set for this problem. The results presented in this article improve and extend some known results according to Long et al. [19], Somyot and Kanokwan [20], and Wang et al. [21].

\section{Preliminary results}

Throughout this article, we suppose that $X, Y$, and $Z$ are real locally convex Hausdorff topological vector spaces, $K \subset X$ and $D \subset Y$ are nonempty compact convex subsets, and $C \subset Z$ is a nonempty closed convex cone. Suppose that $S_{1}, S_{2}: K \times K \rightarrow 2^{K}, T_{1}$, $T_{2}: K \times K \rightarrow 2^{D}$ and $F_{1}, F_{2}: K \times D \times K \rightarrow 2^{Z}$ are set-valued mappings.

For our main results, we need some definitions and lemmas as follows. 
Definition 2.1. Let $X$ and $Y$ be two topological vector spaces and $T: X \rightarrow 2^{Y}$ be a set-valued mapping.

(i) $T$ is said to be upper semicontinuous at $x \in X$ if, for any neighborhood $U$ of $T$ (x), there exists a neighborhood $V$ of $x$ such that $T(t) \subset U$, for all $t \in V$. T is said to be upper semicontinuous on $X$ if it is upper semicontinuous at each $x \in X$.

(ii) $T$ is said to be lower semicontinuous at $x \in X$ if, for any open set $U$ with $T(x) \cap$ $U \neq \varnothing$, there exists a neighborhood $V$ of $x$ such that $T\left(x^{\prime}\right) \cap U \neq \varnothing$, for all $x^{\prime} \in V$. $T$ is said to be lower semicontinuous on $X$ if it is lower semicontinuous at each $x$ $\in X$.

(iii) $T$ is said to be continuous on $X$ if it is both upper semicontinuous and lower semicontinuous on $X$.

(iv) $T$ is said to be closed if, $\operatorname{Graph}(T)=\{(x, y): x \in X, y \in T(x)\}$ is a closed subset in $X \times Y$.

Definition 2.2. Let $W$ be a topological vector space and $D \subset W$ be a nonempty set. A set-valued mapping $G: D \rightarrow 2^{Z}$ is said to be type I $C$-lower semicontinuous at $x_{0}$ if, for any neighborhood $U$ of 0 in $Z$, there exists a neighborhood $U\left(x_{0}\right)$ of $x_{0}$ such that

$$
G\left(x_{0}\right) \subset G(x)+U-C, \quad \forall x \in U\left(x_{0}\right) \cap D .
$$

Definition 2.3. Let $W$ be a topological vector space and $D \subset W$ be a nonempty set. A set-valued mapping $G: D \rightarrow 2^{Z}$ is said to be type II $C$-lower semicontinuous at $x_{0}$ if, for each $z \in G\left(x_{0}\right)$ and any neighborhood $U$ of 0 in $Z$, there exists a neighborhood $U\left(x_{0}\right)$ of $x_{0}$ such that

$$
G(x) \cap(z+U-C) \neq \emptyset, \quad \forall x \in U\left(x_{0}\right) \cap D .
$$

Definition 2.4. Let $W$ be a topological vector space and $D \subset W$ be a nonempty convex set. A set-valued mapping $G: D \rightarrow 2^{Z}$ is said to be $C$-properly quasiconvex if, for any $x, y \in D, t \in[0,1]$, we have

$$
\text { either } G(x) \subset G(t x+(1-t) y)+C \text { or } G(y) \subset G(t x+(1-t) y)+C \text {. }
$$

Definition 2.5. Let $W$ be a topological vector space and $D \subset W$ be a nonempty set. A set-valued mapping $G: D \rightarrow 2^{Z}$ is said to be $C$-convex if, for any $x, y \in D$ and $t \in$ $[0,1]$, one has

$$
G(t x+(1-t) y) \subset t G(x)+(1-t) G(y)-C .
$$

$G$ is said to be $C$-concave if $-G$ is $C$-convex.

Definition 2.6. Let $X$ be a Hausdorff topological vector space and $K \subset X$ be a nonempty set. A set-valued mapping $G: K \rightarrow 2^{X}$ is said to be a KKM mapping if, for any finite set $\left\{x_{1}, \ldots, x_{n}\right\} \subset K$, the relation

$$
\operatorname{co}\left\{x_{1}, \ldots, x_{n}\right\} \subset \bigcup_{i=1}^{n} G\left(x_{i}\right)
$$

holds, where $\operatorname{co}\left\{x_{1}, \ldots, x_{n}\right\}$ denotes the convex hull of $\left\{x_{1}, \ldots, x_{n}\right\}$. 
Lemma 2.1. ([22]) Let $X$ and $Y$ be two Hausdorff topological spaces and $T: X \rightarrow 2^{Y}$ be a set-valued mapping.

(i) If $T$ is upper semicontinuous with closed values, then $T$ is closed.

(ii) If $T$ is closed and $Y$ is compact, then $T$ is upper semicontinuous.

Lemma 2.2. (Kakutani-Fan-Glicksberg [23]) Let $X$ be a locally convex Hausdorff topological vector space and $K$ be a nonempty compact convex subset of $X$. Let $T: K$ $\rightarrow 2^{K}$ be a upper semicontinuous set-valued mapping with nonempty closed convex values. Then, there exists $\bar{x} \in K$ such that $\bar{x} \in T(\bar{x})$.

Lemma 2.3. ([24]) Let $X$ and $Y$ be two Hausdorff topological vector spaces and $T: X$ $\rightarrow 2^{Y}$ be a set-valued mapping with compact values. Then, $T$ is upper semicontinuous on $x \in X$ if and only if for any set $\left\{x_{\alpha}\right\}$ with $x_{\alpha} \rightarrow x$, and $y_{\alpha} \in T\left(x_{\alpha}\right)$, there exists $y \in$ $T(x)$, and a subset $\left\{y_{\beta}\right\}$ of $\left\{y_{\alpha}\right\}$, such that $y_{\beta} \rightarrow y$.

Lemma 2.4. ([25]) Let $D$ be a nonempty convex compact subset of Hausdorff topological vector space $X$ and $E$ be a subset of $D \times D$ such that

(i) for each $x \in D,(x, x) \notin E$;

(ii) for each $x \in D,\{y \in D:(x, y) \in E\}$ is convex;

(iii) for each $y \in D,\{x \in D:(x, y) \in E\}$ is open in $D$.

Then, there exists $\bar{x} \in D$ such that $(\bar{x}, y) \notin E$ for all $y \in D$.

Lemma 2.5. ([26]) Let $K$ be a nonempty subset of a topological vector space $X$ and $F$ $: K \rightarrow 2^{X}$ be a KKM mapping with closed values. Assume that there exists a nonempty compact convex subset $B$ of $K$ such that $D=\bigcap_{x \in B} F(x)$ is compact. Then, $\bigcap_{x \in K} F(x) \neq \emptyset$.

\section{Main results}

In this section, we apply Kakutani-Fan-Glicksberg fixed point theorem to prove an existence theorem of solutions for the SSVQEP. Moreover, we prove the closedness of the solution set for this problem.

Lemma 3.1. Let $W$ be a topological vector space and $D \subset W$ be a nonempty subset. Let $G: D \rightarrow 2^{Z}$ be a set-valued mapping.

(i) If $G$ is lower semicontinuous, then $G$ is type II $C$-lower semicontinuous.

(ii) If $G$ is type I $(-C)$-lower semicontinuous, then $G$ is type II $C$-lower semicontinuous.

(iii) If $G$ is single-valued mapping, then $G$ is type I (-C)-lower semicontinuous $\Leftrightarrow G$ is type II $C$-lower semicontinuous.

Proof. (i) It is easy to verify that the assertion (i) holds and so we omit the proof.

(ii) Suppose that $G$ is type I (-C)-lower semicontinuous at $x_{0} \in D$. Then, for any $z \in$ $G\left(x_{0}\right)$, and for any neighborhood $U$ of 0 in $Z$, there exists a balanced neighborhood $V$ of 0 in $Z$ such that $V \subset U$. Since $G$ is type I (-C)-lower semicontinuous at $x_{0} \in D$, it follows that there exists a neighborhood $U\left(x_{0}\right)$ of $x_{0}$ such that 


$$
G\left(x_{0}\right) \subset G\left(x^{\prime}\right)+V+C, \quad \forall x^{\prime} \in U\left(x_{0}\right) \cap D .
$$

For $z \in G\left(x_{0}\right)$, there exist $y \in G\left(x^{\prime}\right), v \in V, c \in C$ such that $z=y+v+c$, and so

$$
y=z-v-c \in z-V-C=z+V-C \subset z+U-C .
$$

Thus,

$$
G\left(x^{\prime}\right) \cap(z+U-C) \neq \emptyset, \quad \forall x^{\prime} \in U\left(x_{0}\right) \cap D .
$$

It follows that $G$ is type II $C$-lower semicontinuous.

(iii) Suppose that $G$ is type II $C$-lower semicontinuous at $x_{0} \in D$. Then, for any neighborhood $U$ of 0 in $Z$, there exists a balanced neighborhood $V$ of 0 in $Z$ such that $V \subset U$. Since $G$ is type II $C$-lower semicontinuous at $x_{0} \in D$, it follows that there exists a neighborhood $U\left(x_{0}\right)$ of $x_{0}$ such that

$$
G\left(x^{\prime}\right) \subset G\left(x_{0}\right)+V-C, \quad \forall x^{\prime} \in U\left(x_{0}\right) \cap D .
$$

Therefore,

$$
G\left(x_{0}\right) \in G\left(x^{\prime}\right)+V+C \subset G\left(x^{\prime}\right)+U+C, \quad \forall x^{\prime} \in U\left(x_{0}\right) \cap D .
$$

This shows that $G$ is type I (-C)-lower semicontinuous. This completes the proof. The following example shows that the converse of (i) of Lemma 3.1 is not true.

Example 3.1. Let the set-valued mapping $F$ from $R$ into its subsets be defined by $F$ $(0)=[0,1]$ and $F(x)=\{0\}$ for all $x \neq 0$. Let $C=R_{+}=[0,+\infty)$. Then, it is easy to see that $F$ is not lower semicontinuous at 0 . In fact, we can find a point $y_{0}=1 \in F(0)=$ $[0,1]$ and a neighborhood $U\left(y_{0}\right)=\left(\frac{1}{2}, \frac{3}{2}\right)$ of $y_{0}$ such that, for any neighborhood $U(0)$ of 0 , there exist some $x_{0} \neq 0 \in U(0)$ satisfying

$$
F\left(x_{0}\right) \cap U\left(y_{0}\right)=\varnothing \text {. }
$$

This shows that $F$ is not lower semicontinuous at 0 . However, we can show that $F$ is type II $C$-lower semicontinuous at 0 . In fact, for each $y \in F(0)=[0,1]$ and any neighborhood $U$ of 0 in $R$, there exists $\varepsilon_{0}>0$ such that $\left(-\varepsilon_{0}, \varepsilon_{0}\right) \subset U$. Hence, $y+\left(-\varepsilon_{0}, \varepsilon_{0}\right)-C$ $=\left(-\infty, y+\varepsilon_{0}\right)$. It is easy to see that, for any neighborhood $U(0)$ of 0 ,

$$
F(x) \cap\left(-\infty, y+\varepsilon_{0}\right) \neq \emptyset, \quad \forall x \in U(0) .
$$

Thus,

$$
F(x) \cap(y+U-C) \neq \emptyset, \quad \forall x \in U(0),
$$

which shows that $F$ is type II $C$-lower semicontinuous at 0 .

Theorem 3.1. For each $i \in\{1,2\}$, let $S_{i}: K \times K \rightarrow 2^{K}$ be continuous set-valued mappings with nonempty compact convex values and $T_{i}: K \times K \rightarrow 2^{D}$ be upper semicontinuous set-valued mappings with nonempty compact convex values. Let $F_{i}: K \times D \times$ $K \rightarrow 2^{Z}$ be set-valued mappings which satisfy the following conditions:

(i) for all $(x, y) \in K \times D, F_{i}(x, y, x) \subset C$;

(ii) for all $(y, z) \in D \times K, F_{i}(\cdot, y, z)$ are $C$-concave;

(iii) for all $(x, y) \in K \times D, F_{i}(x, y, \cdot)$ are $C$-properly quasiconvex;

(iv) $F_{i}(\cdot, \cdot, \cdot)$ are type II $C$-lower semicontinuous. 
Then, the SSVQEP has a solution. Moreover, the solution set of the SSVQEP is closed.

Proof. For any $(x, y, u, v) \in K \times D \times K \times D$, define mappings $A, B: K \times D \times K \rightarrow 2^{K}$ by

$$
\begin{aligned}
& A(x, y, u)=\left\{a \in S_{1}(x, u): F_{1}(a, y, z) \subset C, \quad \forall z \in S_{1}(x, u)\right\}, \\
& B(x, v, u)=\left\{b \in S_{2}(x, u): F_{2}(b, v, z) \subset C, \quad \forall z \in S_{2}(x, u)\right\} .
\end{aligned}
$$

(I) For any $(x, y, u) \in K \times D \times K, A(x, y, u)$ is nonempty.

Indeed, by the assumption, $S_{1}(x, u)$ is nonempty compact convex set for each $(x, u)$ $\in K \times K$. Set

$$
E=\left\{(a, z) \in S_{1}(x, u) \times S_{1}(x, u): F_{1}(a, y, z) \not \subset C\right\} .
$$

If $E$ is empty, then it is clear that $A(x, y, u)$ is nonempty. Thus, we consider that $E$ is not empty. For any $z \in S_{1}(x, u)$, if $(a, z) \in E$, then there exists $d \in F_{1}(a, y, z)$ such that $d \notin C$. Hence, there exists an open neighborhood $U$ of 0 in $Z$ such that $(d+U) \cap C=$ $\varnothing$ and so

$$
(d+U-C) \cap C=\emptyset .
$$

By the type II $C$-lower semicontinuity of $F_{1}$, there exists an open neighborhood $U_{1}$ of $a$ in $S_{1}(x, u)$ such that

$$
F_{1}\left(a^{\prime}, y, z\right) \cap(d+U-C) \neq \emptyset, \quad \forall a^{\prime} \in U_{1} \cap S_{1}(x, u) .
$$

From (3.2), we know that there exists $d^{\prime} \in F_{1}\left(a^{\prime}, y, z\right)$ such that $d^{\prime} \in d+U-C$. By (3.1), $d^{\prime} \notin C$. Hence,

$$
F_{1}\left(a^{\prime}, y, z\right) \not \subset C, \quad \forall a^{\prime} \in U_{1} \cap S_{1}(x, u) .
$$

Thus, for any $z \in S_{1}(x, z),\left\{a \in S_{1}(x, u):(a, z) \in E\right\}$ is open in $S_{1}(x, u)$. For any $a \in$ $S_{1}(x, u),\left(a, z_{1}\right) \in E,\left(a, z_{2}\right) \in E, t \in[0,1]$, it follows that

$$
F_{1}\left(a, y, z_{1}\right) \not \subset C \quad \text { and } \quad F_{1}\left(a, y, z_{2}\right) \not \subset C .
$$

By condition (iii), we have

$$
\text { either } \quad F_{1}\left(a, y, z_{1}\right) \subset F_{1}\left(a, y, t z_{1}+(1-t) z_{2}\right)+C \quad \text { or } \quad F_{1}\left(a, y, z_{2}\right) \subset F_{1}\left(a, y, t z_{1}+(1-t) z_{2}\right)+C .
$$

We claim that $F_{1}\left(a, y, t z_{1}+(1-t) z_{2}\right) \nsubseteq C$. If not, then by (3.5), we have

$$
\text { either } F_{1}\left(a, y, z_{1}\right) \subset C \text { or } F_{1}\left(a, y, z_{2}\right) \subset C \text {, }
$$

which contradicts (3.4). Hence, $F_{1}\left(a, y, t z_{1}+(1-t) z_{2}\right) \notin C$. Thus, for any $a \in S_{1}(x$, $u),\left\{z \in S_{1}(x, u):(a, z) \in E\right\}$ is convex in $S_{1}(x, u)$. The condition (i) implies that for any $a \in S_{1}(x, u),(a, a) \notin E$. By Lemma 2.4, there exists $a \in S_{1}(x, u)$ such that $(a, z) \notin E$ for all $z \in S_{1}(x, u)$ i.e., $F_{1}(a, y, z) \subset C$ for all $z \in S_{1}(x, u)$. Hence, $A(x, y, u) \neq \varnothing$.

(II) For any $(x, y, u) \in K \times D \times K, A(x, y, u)$ is convex. 
In fact, let $a_{1}, a_{2} \in A(x, y, u), t \in[0,1]$. Then, $a_{1}, a_{2} \in S_{1}(x, u)$,

$$
F_{1}\left(a_{1}, y, z\right) \subset C, \quad \forall z \in S_{1}(x, u)
$$

and

$$
F_{1}\left(a_{2}, y, z\right) \subset C, \quad \forall z \in S_{1}(x, u) .
$$

By the convexity of $S_{1}(x, u)$, we have $t a_{1}+(1-t) a_{2} \in S_{1}(x, y)$. It follows from (3.6) and (3.7) that

$$
F_{1}\left(t a_{1}+(1-t) a_{2}, y, z\right) \subset t F_{1}\left(a_{1}, y, z\right)+(1-t) F_{1}\left(a_{2}, y, z\right)+C \subset C+C+C \subset C, \quad \forall z \in S_{1}(x, u) .
$$

Thus, $t a_{1}+(1-t) a_{2} \in A(x, y, u)$. Therefore, $A(x, y, u)$ is convex.

(III) $A$ is upper semicontinuous on $K \times D \times K$.

Since $K$ is compact, we only need to show that $A$ is a closed mapping. Let $\left\{\left(x_{\alpha}, y_{\alpha}\right.\right.$, $\left.\left.u_{\alpha}\right): \alpha \in I\right\} \subset K \times D \times K$ be a set with $\left(x_{\alpha}, y_{\alpha}, u_{\alpha}\right) \rightarrow(x, y, u) \in K \times D \times K$. Let $v_{\alpha} \in$ $A\left(x_{\alpha}, y_{\alpha}, u_{\alpha}\right)$ with $v_{\alpha} \rightarrow v$. We will show that $v \in A(x, y, u)$. Since $S_{1}$ is upper semicontinuous mapping with nonempty closed values, it follows that $S_{1}$ is a closed mapping. It follows from $v_{\alpha} \in S_{1}\left(x_{\alpha}, u_{\alpha}\right)$ and $\left(x_{\alpha}, u_{\alpha}, v_{\alpha}\right) \rightarrow(x, u, v)$ that we have $v \in S_{1}(x, u)$. Now we claim that $v \in A(x, y, u)$. If not, then there exists $z_{1} \in S_{1}(x, u)$ such that

$$
F_{1}\left(v, y, z_{1}\right) \not \subset C .
$$

Hence, there exists $d \in F_{1}\left(v, y, z_{1}\right)$ such that $d \neq C$, and so there exists an open neighborhood $U$ of 0 in $Z$ such that $(d+U) \cap C+\varnothing$. Therefore,

$$
(d+U-C) \cap C=\emptyset .
$$

Since $F_{1}$ is type II $C$-lower semicontinuous, for $d \in F_{1}\left(v, y, z_{1}\right)$ and $U$, there exists a neighborhood $U\left(v, y, z_{1}\right)$ of $\left(v, y, z_{1}\right)$ such that, for all $F_{1}\left(v^{\prime}, \gamma^{\prime}, z_{1}^{\prime}\right) \cap(d+U-C) \neq \emptyset$.

$$
F_{1}\left(v^{\prime}, \gamma^{\prime}, z_{1}^{\prime}\right) \cap(d+U-C) \neq \emptyset .
$$

Since $\left(x_{\alpha}, u_{\alpha}\right) \rightarrow(x, u)$ and $S$ is lower semicontinuous, for $z_{1} \in S_{1}(x, u)$, there exists $z_{\alpha} \in S_{1}\left(x_{\alpha}, u_{\alpha}\right)$ such that $z_{\alpha} \rightarrow z_{1}$. Thus, $\left(v_{\alpha}, y_{\alpha}, z_{\alpha}\right) \rightarrow\left(v, y, z_{1}\right)$. It follows from (3.10) that there exists $\alpha_{0} \in I$ such that, for $\alpha \geq \alpha_{0}$,

$$
F_{1}\left(v_{\alpha}, \gamma_{\alpha}, z_{\alpha}\right) \cap(d+U-C) \neq \emptyset .
$$

From $v_{\alpha} \in A\left(v_{\alpha}, y_{\alpha}, z_{\alpha}\right)$, we have

$$
F_{1}\left(v_{\alpha}, \gamma_{\alpha}, z\right) \subset C, \quad \forall z \in S_{1}\left(x_{\alpha}, u_{\alpha}\right) .
$$

Because $z_{\alpha} \in S_{1}\left(x_{\alpha}, u_{\alpha}\right)$, we get $F_{1}\left(v_{\alpha}, y_{\alpha}, z_{\alpha}\right) \subset C$. It follows from (3.9) that

$$
F_{1}\left(v_{\alpha}, \gamma_{\alpha}, z_{\alpha}\right) \cap(d+U-C) \subset C \cap(d+U-C)=\emptyset,
$$

which contradicts (3.11). Hence, $A$ is a closed mapping.

Similarly, we know for any $(x, v, u) \in K \times D \times K, B$ is upper semicontinuous on $K \times$ $D \times K$ with nonempty closed convex values. 
(IV) Define the set-valued mappings $H, G: K \times D \times K \rightarrow 2^{K \times D}$ by

$$
H(x, y, u)=\left(A(x, y, u), T_{1}(x, u)\right), \quad \forall(x, y, u) \in K \times D \times K
$$

and

$$
G(x, v, u)=\left(B(x, v, u), T_{2}(x, u)\right), \quad \forall(x, v, u) \in K \times D \times K .
$$

Then, it is easy to see that $H$ and $G$ are upper semicontinuous mappings with nonempty closed convex values.

Define the set-valued mapping $M:(K \times D) \times(K \times D) \rightarrow 2^{(K \times D) \times(K \times D)}$ by

$$
M((x, y),(u, v))=(H(x, y, u), G(x, v, u)), \quad \forall((x, y),(u, v)) \in(K \times D) \times(K \times D) .
$$

Then, we know that $M$ is upper semicontinuous mapping with nonempty closed convex values. By Lemma 2.2 , there exists a point $((\bar{x}, \bar{y}),(\bar{u}, \bar{v})) \in(K \times D) \times(K \times D)$ such that $((\bar{x}, \bar{y}),(\bar{u}, \bar{v})) \in M((\bar{x}, \bar{y}),(\bar{u}, \bar{v}))$, that is

$$
(\bar{x}, \bar{y}) \in H(\bar{x}, \bar{y}, \bar{u}),(\bar{u}, \bar{v}) \in G(\bar{x}, \bar{v}, \bar{u}) .
$$

This implies that $\bar{x} \in A(\bar{x}, \bar{y}, \bar{u}), \quad \bar{y} \in T_{1}(\bar{x}, \bar{u}), \quad \bar{u} \in B(\bar{x}, \bar{v}, \bar{u}), \quad \bar{v} \in T_{2}(\bar{x}, \bar{u})$. Hence, $\bar{y} \in T_{1}(\bar{x}, \bar{u}), \bar{y} \in T_{1}(\bar{x}, \bar{u}), \bar{u} \in S_{2}(\bar{x}, \bar{u}), \bar{v} \in T_{2}(\bar{x}, \bar{u})$,

$$
F_{1}(\bar{x}, \bar{y}, z) \subset C, \quad \forall z \in S_{1}(\bar{x}, \bar{u})
$$

and

$$
F_{2}(\bar{u}, \bar{v}, z) \subset C, \quad \forall z \in S_{2}(\bar{x}, \bar{u}) .
$$

Next, we show that the solution set of the SSVQEP is closed. Let $\left\{\left(x_{\alpha}, u_{\alpha}\right): \alpha \in I\right\}$ be a set in the set of solutions of SSVQEP with $\left(x_{\alpha}, u_{\alpha}\right) \rightarrow(\bar{x}, \bar{u})$, and so there exist $y_{\alpha} \in$ $T_{1}\left(x_{\alpha}, u_{\alpha}\right), v_{\alpha} \in T_{2}\left(x_{\alpha}, u_{\alpha}\right), x_{\alpha} \in S_{1}\left(x_{\alpha}, u_{\alpha}\right), u_{\alpha} \in S_{2}\left(x_{\alpha}, u_{\alpha}\right)$, such that

$$
F_{1}\left(x_{\alpha}, y_{\alpha}, z\right) \subset C, \quad \forall z \in S_{1}\left(x_{\alpha}, u_{\alpha}\right)
$$

and

$$
F_{2}\left(u_{\alpha}, v_{\alpha}, z\right) \subset C, \quad \forall z \in S_{2}\left(x_{\alpha}, u_{\alpha}\right) .
$$

Since $S_{1}$ and $S_{2}$ are upper semicontinuous set-valued mappings with nonempty closed values, it follows from Lemma 2.1 that $S_{1}$ and $S_{2}$ are closed mappings. Thus, $\bar{x} \in S_{1}(\bar{x}, \bar{u})$ and $\bar{u} \in S_{2}(\bar{x}, \bar{u})$. Since $T_{1}$ is upper semicontinuous set-valued mapping with nonempty compact values, by Lemma 2.3 , there exist $\bar{y} \in T_{1}(\bar{x}, \bar{u})$ and a subset $\left\{y_{\beta}\right\}$ of $\left\{y_{\alpha}\right\}$ such that $y_{\beta} \rightarrow \bar{\gamma}$. Similarly, there exist $\bar{v} \in T_{2}(\bar{x}, \bar{u})$ and a subset $\left\{v_{\gamma}\right\}$ of $\left\{v_{\alpha}\right\}$ such that $v_{\gamma} \rightarrow \bar{v}$. By the condition (iv), similar to the proof of the part (III), we have

$$
F_{1}(\bar{x}, \bar{y}, z) \subset C, \quad \forall z \in S_{1}(\bar{x}, \bar{u})
$$

and

$$
F_{2}(\bar{u}, \bar{v}, z) \subset C, \quad \forall z \in S_{2}(\bar{x}, \bar{u}) .
$$

Hence, $(\bar{x}, \bar{u})$ belongs to the set of solutions of SSVQEP. Thus, the set of solutions of SSVQEP is closed set. This completes the proof.

Now we give an example to show Theorem 3.1 is applicable.

Example 3.2. Let $X=Y=Z=R, C=[0,+\infty)$ and $K=D=[0,1]$. For each $x \in K, u$ $\in \quad K$, let $S_{1}(x, u)=\left[\frac{x}{2}, 1\right], \quad S_{2}(x, u)=\left[0, \frac{u+1}{2}\right], \quad T_{1}(x, u)=\left[0, \frac{1-x}{3}+\frac{1-u}{2}\right] \quad$ and 
$F_{1}(x, y, z)=[x+y-z,+\infty), \quad \forall(x, y, z) \in K \times D \times K$. Define the set-valued mappings $F_{1}$ and $F_{2}$ as follows:

$$
F_{1}(x, y, z)=[x+y-z,+\infty), \quad \forall(x, y, z) \in K \times D \times K
$$

and

$$
F_{2}(x, y, z)=[2 x+3 y-z,+\infty), \quad \forall(x, y, z) \in K \times D \times K
$$

It is easy to verify that all the conditions in Theorem 3.1 are satisfied. By Theorem 3.1, we know that SSVQEP has a solution. Let $M$ be the solution set of SSVQEP. Then,

$$
M=\{(\bar{x}, \bar{u}) \in K \times K: 4 \bar{x}-3 \bar{u} \geq 1,3 \bar{u}-3 \bar{x}+2 \geq 0\} .
$$

It is easy to see that $M$ is a closed subset of $K \times K$.

For each $i=\{1,2\}$, if we suppose that $S_{i}$ is a set-valued mapping from $K$ to $K$ and $T_{i}$ is a set-valued mapping from $K$ to $D$, then similar to the proof of Theorem 3.1, we have the following corollary.

Corollary 3.1. Let $S_{1}, S_{2}: K \rightarrow 2^{K}$ be continuous set-valued mappings with nonempty compact convex values and $T_{1}, T_{2}: K \rightarrow 2^{D}$ be upper semicontinuous set-valued mappings with nonempty compact convex values. Let $F_{1}, F_{2}: K \times D \times K \rightarrow 2^{Z}$ be setvalued mappings satisfy the conditions (i)-(iv) of Theorem 3.1. Then, the SGSVQEP has a solution, i.e., there exist $(\bar{x}, \bar{u}) \in K \times K$ and $\bar{y} \in T_{1}(\bar{u}), \bar{v} \in T_{2}(\bar{x})$ such that $\bar{u} \in S_{2}(\bar{u}), \bar{u} \in S_{2}(\bar{u})$

$$
F_{1}(\bar{x}, \bar{y}, z) \subset C, \quad \forall z \in S_{1}(\bar{x})
$$

and

$$
F_{2}(\bar{u}, \bar{v}, z) \subset C, \quad \forall z \in S_{2}(\bar{u}) .
$$

Moreover, the solution set of the SGSVQEP is closed.

Remark 3.1. In [20], Somyot and Kanokwan also obtained an existence result for SGSVQEP. However, the assumptions of Theorem 3.1 in [20] are quite different from the ones in Corollary 3.1. The following example shows the case, where Corollary 3.1 is applicable, but the hypotheses of the corresponding theorem in [20] cannot be satisfied.

Example 3.3. Let $X=Y=Z=R, C=[0,+\infty)$ and $K=D=[0,1]$. For each $x \in K$, $u \in K$, let $S_{1}(x)=[x, 1], S_{2}(x)=\left[\frac{1-x}{2}, \frac{1}{2}\right], T_{1}(x)=[1-x, 1]$ and $T_{2}(x)=\left[0, \frac{1+x}{2}\right]$. Define the set-valued mappings $F_{1}$ and $F_{2}$ as follows:

$$
F_{1}(x, y, z)=\left[2 x+\frac{y}{2}-z,+\infty\right), \quad \forall(x, y, z) \in K \times D \times K
$$

and

$$
F_{2}(x, y, z)=[3 x+y-3 z,+\infty), \quad \forall(x, y, z) \in K \times D \times K .
$$

It is easy to verify that all the conditions in Corollary 3.1 are satisfied. Hence, by Corollary 3.1, SGSVQEP has a solution. Let $N$ be the solution set of SGSVQEP. Then, 


$$
N=\left\{\bar{x}, \bar{u} \in K \times K: \bar{x} \geq \frac{1}{4}, 3 \bar{u}+\frac{\bar{x}}{2}-1 \geq 0\right\} .
$$

It is easy to see that $N$ is a closed subset of $K \times K$. However, the hypothesis (i) of Theorem 3.1 in [20] is not satisfied. Thus, Theorem 3.1 in [20] is not applicable.

If we take $S=S_{1}=S_{2}, F=F_{1}=F_{2}$, and $T=T_{1}=T_{2}$; then, from Corollary 3.1, we have the following corollary.

Corollary 3.2. Let $S: K \rightarrow 2^{K}$ be continuous set-valued mappings with nonempty compact convex values and $T: K \rightarrow 2^{D}$ be upper semicontinuous set-valued mappings with nonempty compact convex values. Let $F: K \times D \times K \rightarrow 2^{Z}$ be set-valued mapping which satisfies the conditions (i)-(iv) of Theorem 3.1. Then, the GSVQEP has a solution, i.e., there exist $\bar{x} \in K$ and $\bar{y} \in T(\bar{x})$ such that $\bar{x} \in S(\bar{x})$ and

$$
F(\bar{x}, \bar{y}, z) \subset C, \quad \forall z \in S(\bar{x}) .
$$

Moreover, the solution set of the GSVQEP is closed.

Remark 3.2. In [19], Long et al. also obtained an existence result for GSVQEP. However, the assumptions in Corollary 3.2 are quite different from the ones in Theorem 3.1 in [19]. The following example shows the case, where Corollary 3.2 is applicable, but the hypotheses of the corresponding theorem in [19] cannot be satisfied.

Example 3.4. Let $X=Y=Z=R, C=[0,+\infty)$ and $K=D=[0,1]$. For each $x \in K$, let $T(x)=\left[0, \frac{x}{2}\right], T(x)=\left[0, \frac{x}{2}\right]$. Define the set-valued mapping $F$ as follows:

$$
F(x, y, z)=[3 x+2 y-z,+\infty), \quad \forall(x, y, z) \in K \times D \times K
$$

It is easy to verify that all the conditions in Corollary 3.2 are satisfied. Hence, by Corollary 3.2, GSVQEP has a solution. Let $O$ be the solution set of GSVQEP. Then,

$$
O=\left\{\bar{x} \in K: \bar{x} \geq \frac{1}{4}\right\} .
$$

It is easy to see that $O$ is a closed subset of $K$. However, the hypothesis (i) of Theorem 3.1 in [19] is not satisfied. Thus, Theorem 3.1 in [19] is not applicable.

If for any $x \in K, S(x)=T(x) \equiv K$ and $F(x, y, z):=F(x, y)$, then GSVQEP collapses to SVEP. Next we give an existence theorem of SVEP on a noncompact set.

Theorem 3.2. Let $X$ and $Z$ be two real Hausdorff topological vector spaces, $K \subset X$ a nonempty closed convex subset and $C \subset Z$ a closed convex cone. Let $F: K \times K \rightarrow 2^{Z}$ be a set-valued mapping. Suppose that

(i) for any $x \in K, F(x, x) \subset C$;

(ii) for any $x \in K$, the set $\{y \in K: F(x, y) \nsubseteq C\}$ is empty or convex;

(iii) for any $y \in K$, the set $\{x \in K: F(x, y) \subset C\}$ is closed;

(iv) there exist a nonempty compact subset $E$ of $K$ and a nonempty convex compact subset $D$ of $K$ such that, for each $x \in K \backslash E$, there exists $y \in D$ such that $F(x, y)$ $\nsubseteq C$.

Then, the SVEP has a solution. Moreover, the solution set of the SVEP is compact.

Proof. We define $G: K \rightarrow 2^{K}$ as follows:

$$
G(y)=\{x \in K: F(x, y) \subset C\} .
$$


It follows from condition (i) that for any $y \in K$, we have $y \in G(y)$ and so $G(y) \neq \varnothing$. We claim that $G$ is a KKM mapping. Suppose to the contrary that there exists a finite subset $\left\{y_{1}, \ldots, y_{n}\right\}$ of $K$, and there exists $\bar{\gamma} \in \operatorname{co}\left\{y_{1}, \ldots, y_{n}\right\}$ such that $\bar{\gamma} \notin \bigcup_{i=1}^{n} G\left(y_{i}\right)$. Hence, $\bar{\gamma}=\sum_{i=1}^{n} t_{i} y_{i}$ for some $t_{i} \geq 0,1 \leq i \leq n$, with $\sum_{i=1}^{n} t_{i}=1$, and $\bar{\gamma} \notin G\left(y_{i}\right)$ for all $i=$ $1, \ldots, n$. Therefore,

$$
F\left(\bar{y}, y_{i}\right) \not \subset C, \quad \forall i=1, \ldots, n .
$$

Equation 3.12 implies that $y_{i} \in\{z \in K, F(\bar{y}, z) \not \subset C\}$, for all $i=1, \ldots, n$. By condition (ii), we have

$$
F(\bar{y}, \bar{y}) \not \subset C,
$$

which contradicts condition (i). Hence, $G$ is a KKM mapping. Applying conditions (iii) and (iv), we deduce that $\bigcap_{y \in D} G(\gamma)$ is a closed subset of $E$. Now, $G$ satisfies all the assumptions of Lemma 2.5 and hence $\bigcap_{y \in K} G(\gamma) \neq \emptyset$. This means that SVEP has a solution. By condition (iii), the solution set of SVEP is closed and by condition (iv), it is subset of the compact set $E$. Thus, the solution set of SVEP is compact. This completes the proof.

Remark 3.3. Theorem 3.2 is different from Theorem 3.1 of Wang et al. [21] in the following two aspects.

(a) The condition (iv) in Theorem 3.2 is weaker than the condition (iv) in Theorem 3.1 of Wang et al. [21]; hence, Theorem 3.2 generalizes Theorem 3.1 of Wang et al. [21];

(b) Theorem 3.2 is proved using Fan-KKM lemma, while Theorem 3.1 of Wang et al. [21] was proved using Brouwer fixed point theorem.

Example 3.5. Let $X=Z=R, K=C=[0,+\infty)$. Define the set-valued mapping $F$ as follows:

$$
F(x, y)=[y-x+1,+\infty), \quad \forall x, y \in K .
$$

If we take $E=[0,1] \cup\{2\}, D=[0,2]$, it is easy to verify that all the conditions in Theorem 3.2 are satisfied. Hence, by Theorem 3.2, SVEP has a solution. Let $P$ be the solution set of SVEP. Then, $P=[0,1]$. It is obvious that $P$ is a compact subset of $K$.

Corollary 3.3. Let $X, Z, K, C$ and $F$ be as in Theorem 3.2. Assume that the conditions (i), (ii), and (iv) of Theorem 3.2 and the following condition holds:

(iii') for any $y \in K, F(\cdot, y)$ is type II $C$-lower semicontinuous.

Then, the SVEP has a solution. Moreover, the solution set of the SVEP is compact.

Proof. By Theorem 3.2, we only need to show that for any $y \in K$, the set

$$
G(y)=\{x \in K: F(x, y) \subset C\}
$$

is closed.

Indeed, let $\left\{x_{\alpha}\right\} \subset G(y)$ be an arbitrary set such that $x_{\alpha} \rightarrow x_{0}$. We need to show that $x_{0} \in G(y)$. Since $x_{\alpha} \in K$ and $K$ is closed, we have $x_{0} \in K$. In addition, for each $\alpha$,

$$
F\left(x_{\alpha}, y\right) \subset C \text {. }
$$

We claim that $F\left(x_{0}, y\right) \subset C$. If not, there exists $z \in F\left(x_{0}, y\right)$ such that $z \notin C$. Hence, there exists a neighborhood $U$ of 0 in $Z$ such that $(z+U) \cap C=\varnothing$, which implies 


$$
(z+U-C) \cap C=\emptyset .
$$

By condition (iii'), we know that there exists $\alpha_{0}$ such that, for $\alpha \geq \alpha_{0}$,

$$
F\left(x_{\alpha}, y\right) \cap(z+U-C) \neq \emptyset .
$$

Hence,

$$
F\left(x_{\alpha}, y\right) \cap(z+U-C) \subset C \cap(z+U-C)=\emptyset,
$$

which contradicts (3.13). Hence, $x_{0} \in G(y)$ and so $G(y)$ is closed. This completes the proof.

Remark 3.4. It follows from Lemma 3.1 that Corollary 3.3 generalizes Theorem 3.2 of Wang et al. [21].

Corollary 3.4. Let $X, Z, K, C$, and $F$ be the same as in Theorem 3.2. Assume that the conditions (i), (iii), and (iv) of Theorem 3.2 and the following condition holds:

(ii') for any $x \in K, F(x, \cdot)$ is $C$-properly quasiconvex.

Then, the SVEP has a solution. Moreover, the solution set of the SVEP is closed.

Proof. By Theorem 3.2, we only need to show that, for any $x \in K$, the set

$$
G(x)=\{y \in K: F(x, y) \not \subset C\}
$$

is convex. Indeed, let $y_{1}, y_{2} \in G(x), t \in[0,1]$,

$$
F\left(x, y_{1}\right) \not \subset C \text { and } F\left(x, y_{2}\right) \not \subset C \text {. }
$$

By condition (iii), we have

$$
\text { either } F\left(x, y_{1}\right) \subset F\left(x, t y_{1}+(1-t) y_{2}\right)+C \text { or } F\left(x, y_{2}\right) \subset F\left(x, t y_{1}+(1-t) y_{2}\right)+C
$$

We claim that $F\left(x, t y_{1}+(1-t) y_{2}\right) \nsubseteq C$. If not, then by (3.15), we have

$$
\text { either } F\left(x, y_{1}\right) \subset C \text { or } F\left(x, y_{2}\right) \subset C \text {, }
$$

which contradicts (3.14). Hence, $F\left(x, t y_{1}+(1-t) y_{2}\right) \mp C$. Thus, $t y_{1}+(1-t) y_{2} \in G(x)$, and so $G(x)$ is convex. This completes the proof.

\footnotetext{
Abbreviations

GSVQEP: generalized strong vector quasiequilibrium problem; SGSVQEP: system of generalized strong vector quasiequilibrium problem; SSVQEP: symmetric strong vector quasiequilibrium problem; SVEP: strong vector equilibrium problem.
}

\section{Acknowledgements}

The authors are grateful to the editor and referees for their valuable comments and suggestions. This study was supported by the Key Program of NSFC (Grant No. 70831005), the National Natural Science Foundation of China (11171237), and the Korea Research Foundation Grant funded by the Korean Government (KRF-2008-313-C00050).

\section{Author details}

${ }^{1}$ Department of Mathematics, Sichuan University, Chengdu, Sichuan 610064, PR China ${ }^{2}$ Department of Mathematics Education and the RINS, Gyeongsang National University, Chinju 660-701, Korea

\section{Authors' contributions}

$\mathrm{BC}$ carried out the study of the existence theorem of solutions and the closedness of the solution set for the symmetric strong vector quasiequilibrium problems and drafted the manuscript. NJH participated in the design of the study and gave some examples to show the main results. YJC conceived of the study, and participated in its design and coordination. All authors read and approved the final manuscript.

\section{Competing interests}

The authors declare that they have no competing interests. 


\section{References}

1. Blum, E, Oettli, W: From optimization and variational inequalities to equilibrium problems. Math Stud. 63, 123-145 (1994)

2. Noor, MA, Oettli, W: On general nonlinear complementarity problems and quasi-equilibria. Mathmatiche. XLIX, 313-331 (1994)

3. Anh, LQ, Khanh, PQ: Existence conditions in symmetric multivalued vector quasiequilibrium problems. Control Cyber. 36, 519-530 (2007)

4. Ansari, QH: Existence of solutions of systems of generalized implicit vector quasi-equilibrium problems. J Math Anal Appl. 341, 1271-1283 (2008). doi:10.1016/j.jmaa.2007.11.033

5. Chen, GY: Existence of solutions for a vector variational inequality: an extension of Hartman-Stampacchia theorem. $J$ Optim Theory Appl. 74, 445-456 (1992). doi:10.1007/BF00940320

6. Fang, YP, Huang, NJ: Feasibility and solvability of vector variational inequalities with moving cones in Banach spaces. Nonlinear Anal. 70, 2024-2034 (2009). doi:10.1016/j.na.2008.02.100

7. Huang, NJ, Fang, YP: On vector variational-like inequalities in reflexive Banach spaces. J Glob Optim. 32, 495-505 (2005) doi:10.1007/s10898-003-2686-z

8. Tan, NX: On the existence of solutions of quasivariational inclusion problems. J Optim Theory Appl. 123, 619-638 (2004). doi:10.1007/s10957-004-5726-z

9. Ansari, QH, Konnov, IV, Yao, JC: Existence of a solution and variational principles for vector equilibrium problems. J Optim Theory Appl. 110, 481-492 (2001). doi:10.1023/A:1017581009670

10. Chen, GY, Huang, XX, Yang, XQ: Vector Optimization: Set-Valued and Variational Analysis. In Lecture Notes in Economics and Mathematical Systems, vol. 541,Springer, Berlin (2005)

11. Fu, JY: Symmetric vector quasiequilibrium problems. J Math Anal Appl. 285, 708-713 (2003). doi:10.1016/S0022-247X(03) 00479-7

12. Gong, XH: Symmetric strong vector quasi-equilibrium problems. Math Methods Oper Res. 65, 305-314 (2007). doi:10.1007/s00186-006-0114-0

13. Hou, SH, Gong, XH, Yang, XM: Existence and stability of solutions for generalized strong vector equilibrium problems with trifunctions. J Optim Theory Appl. 146, 387-398 (2010). doi:10.1007/s10957-010-9656-7

14. Huang, NJ, Li, J, Yao, JC: Gap functions and existence of solutions to a system of vector equilibrium problems. J Optim Theory Appl. 133, 201-212 (2007). doi:10.1007/s10957-007-9202-4

15. Kimura, K, Yao, JC: Semicontinuity of solution mappings of parametric generalized vector equilibrium problems. J Optim Theory Appl. 138, 429-443 (2008). doi:10.1007/s10957-008-9386-2

16. Ansari, QH, Oettli, W, Schiager, D: A generalization of vectorial equilibria. In Proceedings of the 2nd International Symposium on Operations Research and Its Applications (ISORA'96), vol. 1114, pp. 181-185.World Publishing, Guilin, China (1996)

17. Jameson, G: Order Linear Spaces. In Lecture Notes in Mathematics, vol. 141,Springer, Berlin (1970)

18. Gong, XH: Strong vector equilibrium problems. J Glob Optim. 36, 339-349 (2006). doi:10.1007/s10898-006-9012-5

19. Long, XJ, Huang, NJ, Teo, KL: Existence and stability of solutions for generalized strong vector quasi-equilibrium problem. Math Comput Model. 47, 445-451 (2008). doi:10.1016/..mcm.2007.04.013

20. Somyot, P, Kanokwan, S: On the existence result for system of generalized strong vector quasiequilibrium problems. Fixed Point Theory Appl

21. Wang, SH, Li, QY, Fu, JY: Strong vector equilibrium problems on noncompact sets. Bull Malays Math Sci Soc. http:// www.emis.de/journals/BMMSS/pdf/acceptedpapers/2009-11-022-R1.pdf (2009)

22. Aubin, JP, Ekeland, I: Applied Nonlinear Analysis. Wiley, New York (1984)

23. Holmes, RB: Geometric Function Analysis and its Application. Springer, New York (1975)

24. Ferro, F: Optimization and stability results through cone lower semicontinuity. Set-valued Anal. 5, 365-375 (1997). doi:10.1023/A:1008653120360

25. Fan, K: A generalization of Tychonoff's fixed point theorem. Math Ann. 142, 303-310 (1961)

26. Fan, K: Some properties of convex sets related to fixed point theorems. Math Ann. 266, 519-537 (1984). doi:10.1007/ BF01458545

doi:10.1186/1029-242X-2011-56

Cite this article as: Chen et al:: Symmetric strong vector quasiequilibrium problems in Hausdorff locally convex

spaces. Journal of Inequalities and Applications 2011 2011:56.

\section{Submit your manuscript to a SpringerOpen ${ }^{\circ}$ journal and benefit from:}

- Convenient online submission

- Rigorous peer review

- Immediate publication on acceptance

- Open access: articles freely available online

- High visibility within the field

- Retaining the copyright to your article

Submit your next manuscript at $>$ springeropen.com 